\title{
REMARKS ON THE ZEROS OF THE ASSOCIATED LEGENDRE FUNCTIONS WITH INTEGRAL DEGREE
}

\author{
J.F. VAN DIEJEN
}

\begin{abstract}
We present some formulas for the computation of the zeros of the integral-degree associated Legendre functions with respect to the order.
\end{abstract}

\section{INTRODUCTION}

The associated Legendre functions (or spherical functions) are given explicitly by AS, GR.

$$
\begin{aligned}
\Gamma(1-z) P_{n}^{z}(\tanh (x)) & =\left(\frac{1+\tanh (x)}{1-\tanh (x)}\right)^{z / 2} F\left(-n, n+1 ; 1-z ; \frac{1-\tanh (x)}{2}\right), \\
& =\frac{\exp (z x)}{\left(1+e^{-2 x}\right)^{n}} F\left(-n,-n-z ; 1-z ;-e^{-2 x}\right),
\end{aligned}
$$

where $\Gamma(-)$ refers to the Euler gamma function and $F(-,-;-;-)$ to the Gauss hypergeometric series, and where we have fixed the normalization such that no gamma factors appear in front of the hypergeometric series representation.

When the degree $n$ is integral the hypergeometric series on the first line of Eq. (1.1) terminates, whence it is then polynomial in the argument $\tanh (x)$ and - with the present normalization - rational in the order $z$. The purpose of the present note is to characterize the locations of zeros of the integral-degree associated Legendre functions with respect to the order $z$. In Refs. [DD1, DD2] detailed information concerning the locations of the zeros of the associated Legendre functions with respect to the argument was provided for the situation that the order and the degree differ by a positive integer. Previously, a numerical study of the locations of the zeros with respect to the degree (for integral order and various values of the argument) was presented in Ref. [B].

Questions about the zeros of the associated Legendre functions fit within a rich tradition of research concerning the locations of the zeros of orthogonal polynomials [S. I]. An important difference is, however, that in the present context we are not in the position to exploit an orthogonality structure. Instead, the idea behind the methods below is to employ a connection with the (inverse) scattering theory of the one-dimensional Schrödinger equation with Pöschl-Teller potential [F], which reveals that the integral-degree associated Legendre functions correspond to reflectionless wave functions that can be expressed in terms of the tau functions of the Korteweg-de Vries hierarchy [DK]. Detailed information on the zeros can then be obtained from Refs. D, DP, where the behavior of the zeros of such reflectionless wave functions was studied with the aid of (Ruijsenaars-Schneider type) integrable

Date: February 2007.

Work supported in part by the 'Anillo Ecuaciones Asociadas a Reticulados', financed by the World Bank through the 'Programa Bicentenario de Ciencia y Tecnología'. 
particle systems (cf. also Ref. [ELZ], where information on the zeros of reflectionless Schrödinger wave functions was obtained via inverse scattering techniques).

This note is organized as follows. First we present a system of algebraic equations for the zeros of the associated Legendre function in Section 2 Next, it is shown in Section 3 that these algebraic equations imply a nonlinear system of coupled differential equations. The solution of these differential equations formulated in Section 4 then provides us with explicit information on the locations of the zeros of the integral-degree associated Legendre functions.

\section{Algebraic Equations}

Let us abbreviate the renormalized associated Legendre function in Eq. (1.1) by $\psi_{n}(x, z)$. Upon writing the hypergeometric series explicitly:

$$
\psi_{n}(x, z)=\frac{\exp (z x)}{\left(1+e^{-2 x}\right)^{n}} \sum_{m=0}^{n} e^{-2 m x}\left(\begin{array}{c}
n \\
m
\end{array}\right) \prod_{j=1}^{m} \frac{z+n+1-j}{z-j},
$$

it is seen that the function in question admits a factorization of the form

$$
\psi_{n}(x, z)=\frac{\exp (z x)}{\left(1+e^{-2 x}\right)^{n}} \prod_{j=1}^{n} \frac{z-z_{j}(x)}{z-j} .
$$

The following proposition provides a (Bethe type) system of algebraic equations for the zeros $z_{1}(x), \ldots, z_{n}(x)$.

Proposition 1 (Algebraic System). The zeros $z_{1}(x), \ldots, z_{n}(x)$ of the normalized integral-degree associated Legendre function $\psi_{n}(x, z)=\Gamma(1-z) P_{n}^{z}(\tanh (x))$ satisfy the rational system

$$
\prod_{j=1}^{n} \frac{\ell-z_{j}(x)}{\ell+z_{j}(x)}=(-1)^{n-\ell} \exp (-2 \ell x), \quad \ell=1, \ldots, n .
$$

Proof. It follows from the Gauss hypergeometric equation (and can also be inferred directly with the aid of Eq. (2.1) ) that the associated Legendre function $\psi_{n}(x, z)$ solves the Schrödinger equation on the line with Pöschl-Teller potential

$$
\left(\frac{\mathrm{d}^{2}}{\mathrm{~d} x^{2}}+\frac{n(n+1)}{\cosh ^{2}(x)}-z^{2}\right) \psi_{n}(x, z)=0 .
$$

From Eq. (2.1) it is manifest that $\psi_{n}(x, z) \rightarrow \exp (z x)$ for $x \rightarrow+\infty$ and $\psi_{n}(x, z) \rightarrow$ $\exp (z x) \prod_{j=1}^{n} \frac{z+j}{z-j}$ for $x \rightarrow-\infty$. In other words, $\psi_{n}(x, z)$ provides a Jost solution of the Schrödinger eigenvalue problem in question and the pertinent Pöschl-Teller potential $n(n+1) / \cosh ^{2}(x)$ is reflectionless. We read-off from the asymptotics for $x \rightarrow \pm \infty$ that the bound states correspond to the spectral values $z=-j$, $j=1, \ldots, n$; the associated normalization constants are given explicitly by (cf. GR, Eqs. (7.122.1), (8.737.1,2)])

$$
\nu_{j}=\left(\int_{-\infty}^{\infty} \psi_{n}^{2}(x,-j) \mathrm{d} x\right)^{-1}=j\left(\begin{array}{c}
2 j \\
j
\end{array}\right)\left(\begin{array}{c}
n+j \\
n-j
\end{array}\right)=(-1)^{n-j} 2 j \prod_{k=1, k \neq j}^{n} \frac{j+k}{j-k} .
$$


The proposition now follows upon specialization of a system of Bethe type equations for the zeros of reflectionless Jost functions derived in Refs. [D, DP]

$$
2 \kappa_{\ell} \prod_{j=1}^{n} \frac{\kappa_{\ell}-z_{j}(x)}{\kappa_{\ell}+z_{j}(x)} \prod_{j=1, j \neq \ell}^{n} \frac{\kappa_{\ell}+\kappa_{j}}{\kappa_{\ell}-\kappa_{j}}=\nu_{\ell} \exp \left(-2 x \kappa_{\ell}\right), \quad \ell=1, \ldots, n,
$$

where $-\kappa_{1}, \ldots,-\kappa_{n}$ represesent the spectral values parametrizing the discrete spectrum and $\nu_{1}, \ldots, \nu_{n}$ denote the values of the associated normalization constants (so in our case $\kappa_{j}=j$ and $\nu_{j}$ is given by the expression in Eq. (2.4)).

\section{Differential Equations}

By differentiating the algebraic equations in Proposition 1, one arrives at a (Dubrovin type) system of first-order differential equations for the zeros.

Proposition 2 (First-order System). The zeros $z_{1}(x), \ldots, z_{n}(x)$ of the normalized integral-degree associated Legendre function $\psi_{n}(x, z)=\Gamma(1-z) P_{n}^{z}(\tanh (x))$ satisfy the system of first-order differential equations

$$
z_{\ell}^{\prime}(x)=\frac{\prod_{j=1}^{n}\left(j^{2}-z_{\ell}^{2}(x)\right)}{\prod_{j=1, j \neq \ell}^{n}\left(z_{j}^{2}(x)-z_{\ell}^{2}(x)\right)}, \quad \ell=1, \ldots, n,
$$

with the initial condition

$$
\left\{\lim _{x \rightarrow 0} z_{1}(x), \ldots, \lim _{x \rightarrow 0} z_{n}(x)\right\}=\{n-1, n-3, \ldots,-(n-3),-(n-1)\} .
$$

Proof. When taking the (logarithmic) derivative of the algebraic equations in Proposition 11, one arrives at the identities

$$
\sum_{j=1}^{n} \frac{z_{j}^{\prime}(x)}{\ell^{2}-z_{j}^{2}(x)}=1, \quad \ell=1, \ldots, n .
$$

These identities may be thought of as a system of $n$ linear equations for the derivatives $z_{1}^{\prime}(x), \ldots, z_{n}^{\prime}(x)$. Solving this linear system leads to the differential equations stated in the proposition. For $x=0$, the hypergeometric series in the second line of Eq. (1.1) simplifies:

$$
\psi_{n}(0, z)=\Gamma(1-z) P_{n}^{z}(0)=2^{-n} F(-n,-n-z ; 1-z ;-1)=\prod_{j=1}^{n} \frac{z+n+1-2 j}{z-j},
$$

whence for $x \rightarrow 0$ the zeros of the integral-degree associated Legendre function tend to $n+1-2 j, j=1, \ldots, n$.

Remark. Upon substituting the product representation of the form in Eq. (2.2) into the Schrödinger equation with Pöschl-Teller potential in the proof of Proposition 1 it is seen that the zeros of the normalized integral-degree associated Legendre function $\psi_{n}(x, z)=\Gamma(1-z) P_{n}^{z}(\tanh (x))$ also satisfy the following system of (Ruijsenaars-Schneider type) second-order differential equations (cf. Refs. [D, DP])

$$
z_{\ell}^{\prime \prime}(x)+2 z_{\ell}(x) z_{\ell}^{\prime}(x)=\sum_{j=1, j \neq \ell}^{n} \frac{2 z_{\ell}^{\prime}(x) z_{j}^{\prime}(x)}{z_{\ell}(x)-z_{j}(x)}, \quad \ell=1, \ldots, n .
$$


From Proposition 2 it follows that the corresponding initial conditions are given by

$$
\begin{aligned}
& z_{\ell}(0)=n+1-2 \ell, \\
& z_{\ell}^{\prime}(0)=\frac{-\prod_{\substack{j=1 \\
j \neq|n+1-2 \ell|}}^{n}\left((n+1-2 \ell)^{2}-j^{2}\right)}{2^{n-1} \prod_{\substack{j=1 \\
j \neq \ell, n+1-\ell}}^{n} 2(\ell-j)^{2}},
\end{aligned}
$$

$\ell=1, \ldots, n$ (where we have ordered the zeros from large to small).

\section{TRAJECTORIES}

We conclude by providing a characterization of the zeros of the associated Legendre functions in terms of the eigenvalues of an explicit square matrix $\mathbf{Z}$ of size equal to the degree $n$.

Proposition 3 (Trajectories). The zeros $z_{1}(x), \ldots, z_{n}(x)$ of the normalized integraldegree associated Legendre function $\psi_{n}(x, z)=\Gamma(1-z) P_{n}^{z}(\tanh (x))$ are given by the (real) eigenvalues of the matrix

$$
\mathbf{Z}(x):=\mathbf{K}(\mathbf{I}-\exp (-2 x \mathbf{K}) \mathbf{N})(\mathbf{I}+\exp (-2 x \mathbf{K}) \mathbf{N})^{-1},
$$

where $\mathbf{K}:=\operatorname{diag}(1,2, \ldots, n), \mathbf{N}$ is the $n \times n$ matrix with components

$$
\mathbf{N}_{j, k}:=\left(\begin{array}{c}
2 j \\
j
\end{array}\right)\left(\begin{array}{c}
n+j \\
n-j
\end{array}\right) \frac{j}{j+k}, \quad 1 \leq j, k \leq n
$$

(and $\mathbf{I}$ represents the $n$-dimensional identity matrix).

Proof. Let $\tilde{\mathbf{Z}}:=\operatorname{diag}\left(z_{1}(x), \ldots, z_{n}(x)\right)$ and let $\mathbf{U}$ denote the orthogonal matrix with components

$$
\mathbf{U}_{j, k}:=\left(\frac{\prod_{\ell=1}^{n}\left(\ell^{2}-z_{j}^{2}(x)\right)}{\prod_{\ell=1, \ell \neq j}^{n}\left(z_{\ell}^{2}(x)-z_{j}^{2}(x)\right)}\right)^{\frac{1}{2}} \frac{1}{z_{j}^{2}(x)-k^{2}}\left(\frac{\prod_{\ell=1}^{n}\left(k^{2}-z_{\ell}^{2}(x)\right)}{\prod_{\ell=1, \ell \neq k}^{n}\left(k^{2}-\ell^{2}\right)}\right)^{\frac{1}{2}},
$$

$1 \leq j, k \leq n$. Then the matrix

$$
\tilde{\mathbf{N}}:=\left(\mathbf{I}-\mathbf{K}^{-1 / 2} \mathbf{U}^{-1} \tilde{\mathbf{Z}} \mathbf{U} \mathbf{K}^{-1 / 2}\right)\left(\mathbf{I}+\mathbf{K}^{-1 / 2} \mathbf{U}^{-1} \tilde{\mathbf{Z}} \mathbf{U} \mathbf{K}^{-1 / 2}\right)^{-1}
$$

has components

$$
\tilde{\mathbf{N}}_{j, k}=\frac{\tilde{\nu}_{j}^{1 / 2} \tilde{\nu}_{k}^{1 / 2}}{j+k}, \quad 1 \leq j, k \leq n,
$$

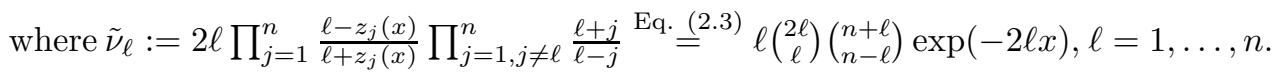
By inverting the relation between $\tilde{\mathbf{Z}}$ and $\tilde{\mathbf{N}}$ it is readily seen that the zeros $z_{1}(x), \ldots, z_{n}(x)$ are given by the eigenvalues of the symmetric matrix

$$
\mathbf{K}^{1 / 2}(\mathbf{I}-\tilde{\mathbf{N}})(\mathbf{I}+\tilde{\mathbf{N}})^{-1} \mathbf{K}^{1 / 2},
$$

which differs from the matrix $\mathbf{Z}(x)$ formulated in the proposition by a diagonal similarity transformation. 
When $x$ runs from $-\infty$ to $+\infty$ along the real axis the argument $y=\tanh (x)$ of the associated Legendre function $P_{n}^{z}(y)$ varies from -1 to 1 . It is clear from Proposition 3 that its zeros $\left\{z_{1}(x), \ldots, z_{n}(x)\right\}$ move in this situation from $\{-1,-2, \ldots,-n\}$ to $\{1,2, \ldots, n\}$. It moreover follows from the general analyis in Ref. [DP] that the corresponding trajectories of the zeros are analytic in $x$ and strictly monotonously increasing in such a way that crossings do not occur (i.e. the zeros remain simple for all $x \in \mathbb{R})$. To illustrate this state of affairs, in Figures 1 and 2 the trajectories of the zeros are plotted for $n=5$ as function of $x$ and of $y=\tanh (x)$, respectively.

Remark. It is manifest from Eq. (2.2) and Proposition 3 that the integral-degree associated Legendre function admits the following determinantal representation

$$
\Gamma(1-z) P_{n}^{z}(\tanh (x))=\frac{e^{z x} \operatorname{det}(z \mathbf{I}-\mathbf{Z}(x))}{\left(1+e^{-2 x}\right)^{n} \prod_{j=1}^{n}(z-j)},
$$

with the matrix $\mathbf{Z}(x)$ as defined in Proposition 3, or equivalently:

$$
\begin{aligned}
& \operatorname{det}(z \mathbf{I}-\mathbf{Z}(x))= F\left(-n,-n-z ; 1-z ;-e^{-2 x}\right) \prod_{j=1}^{n}(z-j) \\
&= \sum_{m=0}^{n} e^{-2 m x}\left(\begin{array}{c}
n \\
m
\end{array}\right) \prod_{j=1}^{m}(z+n+1-j) \prod_{k=m+1}^{n}(z-k) .( \\
& \text { REFERENCES }
\end{aligned}
$$

\section{REFERENCES}

AS. M. Abramowitz and I.A. Stegun (eds.), Handbook of Mathematical Functions with Formulas, Graphs, and Mathematical Tables, Dover Publications, New York, 1972.

B. H.F. Bauer, Tables of the roots of the associated Legendre function with respect to the degree, Math. Comp. 46 (1986), no. 174, 601-602, S29-S41.

D. J.F. van Diejen, On the zeros of the KdV soliton Baker-Akhiezer function, Regul. Chaotic Dyn. 4 (1999), no. 2, 103-111.

DK. J.F. van Diejen and A.N. Kirillov, A combinatorial formula for the associated Legendre functions of integer degree, Adv. Math. 149 (2000), no. 1, 61-88.

DP. J.F. van Diejen and H. Puschmann, Reflectionless Schödinger operators, the dynamics of zeros, and the solitonic Sato formula, Duke Math. J. 104 (2000), no. 2, 269-318.

DD1. K. Driver and P. Duren, Zeros of the hypergeometric polynomials $F(-n, b ; 2 b ; z)$, Indag. Math. (N.S.) 11 (2000), no. 1, 43-51.

DD2. Trajectories of the zeros of hypergeometric polynomials $F(-n, b ; 2 b ; z)$ for $b<-\frac{1}{2}$, Constr. Approx. 17 (2001), no. 2, 169-179.

ELZ. N.M. Ercolani, C.D. Levermore, and T. Zhang, The behavior of the Weyl function in the zero-dispersion KdV limit, Comm. Math. Phys. 183 (1997), no. 1, 119-143.

F. S. Flügge, Practical Quantum Mechanics, Classics in Mathematics, Springer-Verlag, Berlin, 1999.

GR. I.S. Gradshteyn and I.M. Ryzhik, Table of Integrals, Series, and Products, fifth edition, Academic Press, Boston, 1994.

I. M.E.H. Ismail, Classical and Quantum Orthogonal Polynomials in One Variable, Encyclopedia of Mathematics and its Applications, Vol. 98, Cambridge University Press, Cambridge, 2005.

S. G. Szegö, Orthogonal Polynomials, fourth edition, American Mathematical Society, Colloquium Publications, Vol. XXIII, American Mathematical Society, Providence, R.I., 2003.

Instituto de Matemática y Física, Universidad de Talca, Casilla 747, Talca, Chile 


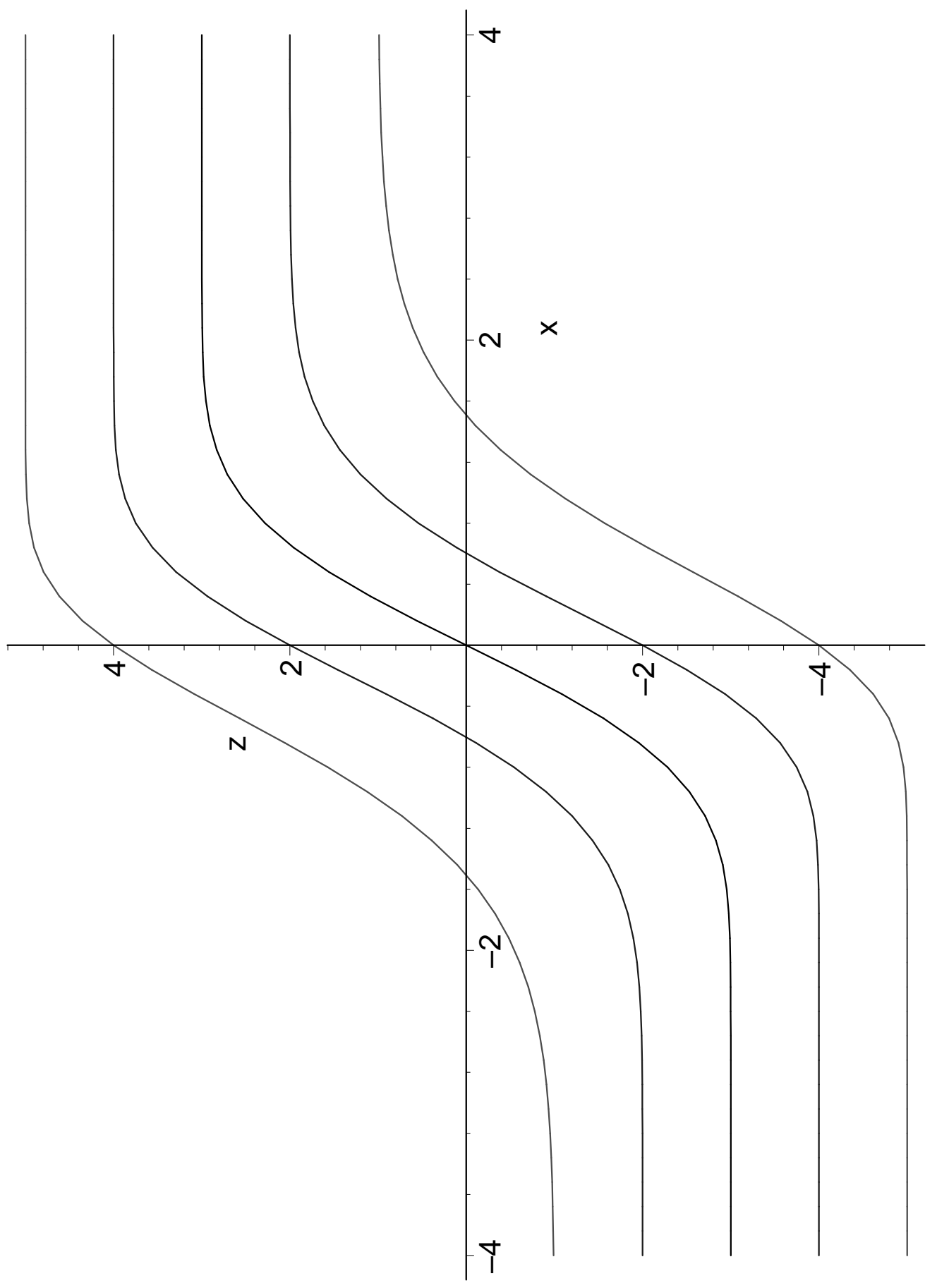

Figure 1. Trajectories of the zeros of $\Gamma(1-z) P_{n}^{z}(\tanh (x))$ with respect to $z$ as a function of $x \in \mathbb{R}$ for $n=5$. 


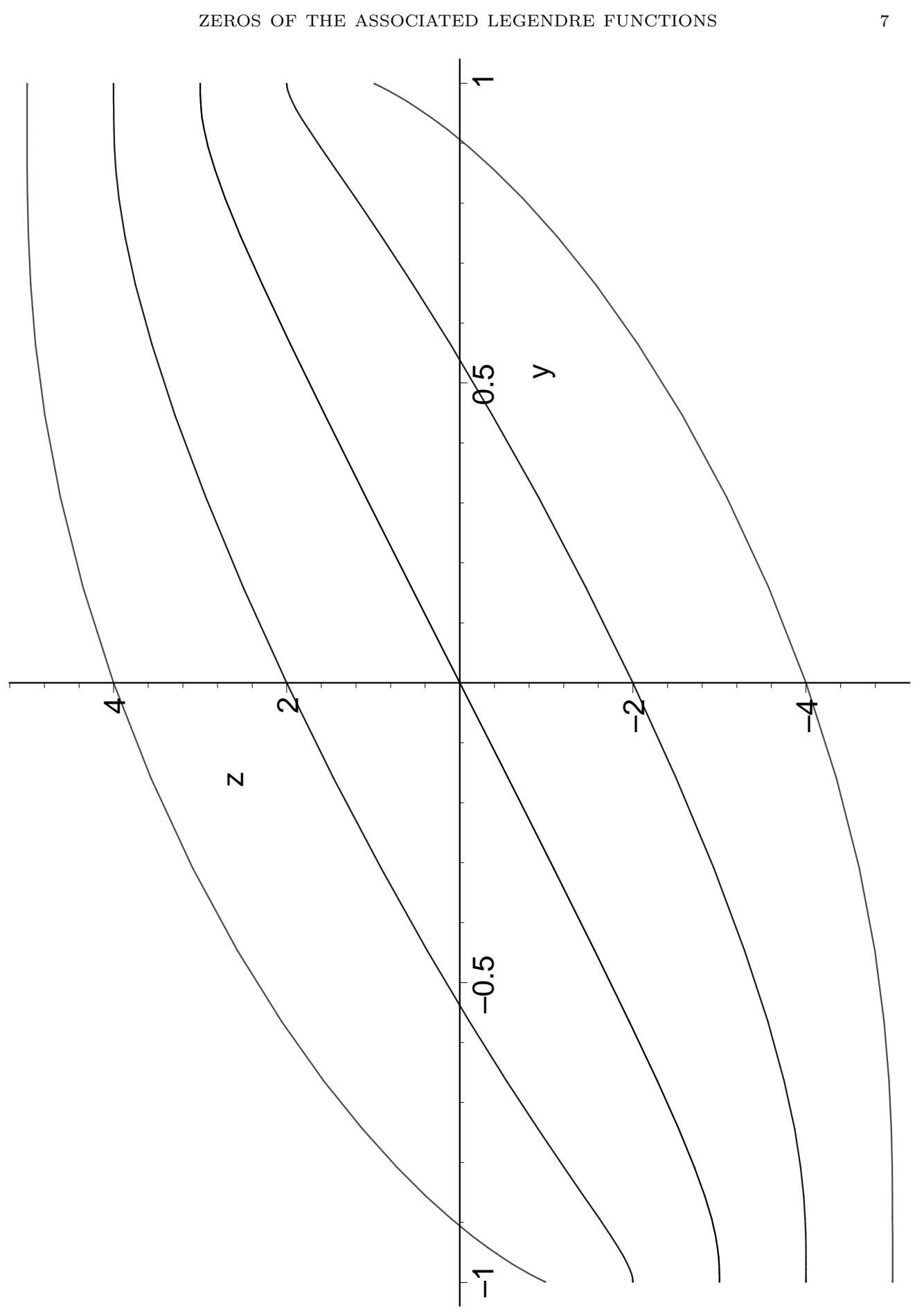

Figure 2. Trajectories of the zeros of $\Gamma(1-z) P_{n}^{z}(y)$ with respect to $z$ as a function of $-1 \leq y \leq 1$ for $n=5$. 